\title{
Calibration of a Polarimetric Radar Using a Rotatable Dihedral Corner Reflector
}

\author{
C. M. H. Unal, R. J. Niemeijer, J. S. van Sinttruyen, and L. P. Ligthart
}

\begin{abstract}
Based on the existing mathematical formalisms of radar polarimetry, it is necessary to perform accurate and diversified polarimetric measurements in the real world to thoroughly investigate signature definition, identification, and classification of radar targets. For this study the Delft Atmospheric Research Radar (DARR) is used. This ground-based polarimetric FM-CW radar operates in the $S$-band. The purpose of the present paper is the polarimetric calibration of the DARR. Among the passive reflectors, a rotatable dihedral corner reflector is a suitable calibration object. It enables one to measure different scattering matrices with only one reflector. One alignment must be performed and the scattering matrices are measured at the same range. By measuring several scattering matrices, the accuracy of the calibration result can be estimated. A measurement campaign with a rotatable dihedral corner reflector was therefore performed. The experimental results and the calibration procedure are presented in this paper.
\end{abstract}

\section{INTRODUCTION}

$\mathrm{T}$ HE DARR is intended for use to gain insight into polarimetric characterization of radar targets and in studies of tropospheric phenomena. The current real-time output is the scattering matrix and the Doppler velocity per range cell. For precipitation, the differential reflectivity $Z_{d r}$, the linear depolarization ratio $L_{d r}$, the horizontal reflectivity $Z_{h}$, and the Doppler spectrum are measured.

Special features of the system are the separate transmitter and receiver antennas specifically designed to provide the necessary high degree of isolation, and dedicated transmitter and receiver polarizers to give the system polarimetric measurement capability, and the sinusoidal steering of the separate polarizers, which makes it possible to determine the relative scattering matrix with a single receive channel. The radar is presented in [1] and [2] with [2] describing in detail the calculation of the scattering matrix and the Doppler velocity. A simplified block diagram is given in Fig. 1 and the specifications of the radar are in Table $\mathbf{I}$. DARR was first used for groundbased remote sensing of precipitation [3], [4].

For the polarimetric calibration of the radar a rotatable dihedral corner reflector is used. When the reflector rotates around the line of sight (the line between the radar and the target), the scattering matrix changes. Therefore, several different scattering matrices can be measured and

Manuscript received December 13, 1993; revised March 22, 1994. This work was supported by The Netherlands Technology Foundation.

The authors are with the Laboratory for Telecommunication and Remote Sensing Technology, Delft University of Technology, 2600 GA Delft, The Netherlands.

IEEE Log Number 9402664.

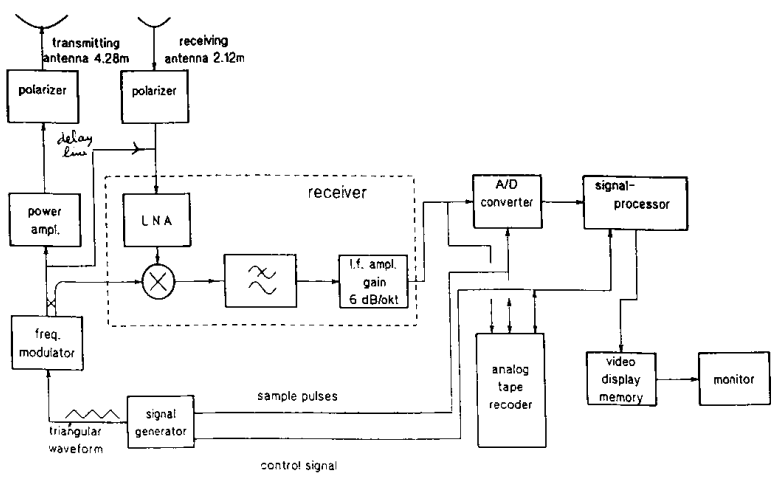

Fig. 1. Simplified block diagram of DARR.

TABLE I

Characteristics of the Delft Atmospheric Research Radar

\begin{tabular}{|ll|}
\hline \multicolumn{2}{c|}{ DARR specifications } \\
\hline Radar type & $\begin{array}{l}\text { linear FM } \\
\text { triangular, sawtooth }\end{array}$ \\
Transmitted power & $30 \mathrm{dBm}(\mathrm{max})$. \\
Center frequency & $3.315 \mathrm{GHz}$ \\
Frequency excursion & $1-50 \mathrm{MHz}$ \\
Range resolution & $3-150 \mathrm{~m}$ \\
Sweep time T & $0.625-640 \mathrm{~ms}$ \\
Beat frequencies & $0.4-1000 \mathrm{kHz}$ \\
Receiver noise figure & $2.5 \mathrm{~dB}$ \\
Antenna gain & $32.7 \mathrm{~dB}$ receiver \\
& $40.0 \mathrm{~dB}$ transmitter \\
Antenna beamwidth & $3.6^{\circ} \mathrm{receiver}$ \\
& $1.6^{\circ} \mathrm{transmitter}$ \\
Antenna isolation & $>90 \mathrm{~dB}$ \\
Range & $0.4-30 \mathrm{~km}$ \\
Analyzer bandwidth B & $1 / \mathrm{T}$ \\
Minimum detectable signal & $-160+10 \mathrm{log}(\mathrm{B}) \mathrm{dBm}$ \\
Fixed target suppression & $>20 \mathrm{~dB}$ \\
Maximum Doppler velocity & $\pm 18 \mathrm{~m} / \mathrm{s}$ \\
Doppler resolution cell & $0.05 \mathrm{~m} / \mathrm{s}$ (minimum) \\
Polarization angles & $\pm 90^{\circ}$ \\
Polarization accuracy & transmitter, receiver \\
Polarizer period & $1.5^{\circ}$ \\
\hline
\end{tabular}

the "combined" antenna distortion matrix (result of the calibration) is retrieved. The system of equations to solve is nonlinear and redundant. The antenna distortion matrix is a least-squares solution the accuracy of which depends on the number of measurements. 


\section{Radar Calibration}

For an ideal polarimetric radar, the measured scattering matrix $Z$ of a target is equal to its theoretical (or actual) scattering matrix $S$. Because this is rarely the case, the errors introduced by the radar antenna must be determined, and then the process must be inverted to obtain an estimate of the actual scattering matrix. The received scattered electric field is expressed in the equations below [5]. The so-called polarization state ( $\vec{e}_{t}$ for transmission and $\vec{e}_{r}$ for reception) is the normalized electric field [6]. The modulus of the polarization state is then equal to 1 . The transmitted electric field expression is modified in amplitude and phase through the action of the transmit antenna distortion matrix $T$. Then, after scattering, the polarization of the scattered electric field at the receiver changes through the action of the matrix $R$, the distortion matrix of the receive antenna.

$$
\begin{aligned}
\vec{E}_{s} & =Z \vec{e}_{t} \\
\vec{E}_{s} & =\frac{K}{r^{2}} e^{-2 j k r}\left[\begin{array}{ll}
R_{h h} & R_{h v} \\
R_{v h} & R_{v v}
\end{array}\right]\left[\begin{array}{ll}
S_{h h} & S_{h v} \\
S_{v h} & S_{v v}
\end{array}\right]\left[\begin{array}{ll}
T_{h h} & T_{h v} \\
T_{v h} & T_{v v}
\end{array}\right] \vec{e}_{t}
\end{aligned}
$$

with

$$
K=\left(\frac{2 \eta_{0} P_{t} G_{t} G_{r} \lambda^{2}}{(4 \pi)^{2}}\right)^{1 / 2}
$$

where

$S$ actual scattering matrix

$Z$ measured scattering matrix

$T$ transmit antenna distortion matrix

$R$ receive antenna distortion matrix

$\vec{e}_{t} \quad$ polarization state of the transmit antenna

$\vec{E}_{s}$ received scattered electric field

$r$ distance radar target or range

$k$ modulus of the propagation vector

$P_{t}$ transmit power

$G_{t}$ gain of the transmit antenna

$G_{r}$ gain of the receive antenna

$\lambda$ radar wavelength

$\eta_{0}$ intrinsic impedance of free space

The received voltage is given in (4) where $\vec{e}_{r}^{T}$ is the transpose of the polarization state of the receive antenna and where the notations of [6] are used.

$$
V=\vec{e}_{r}^{T} \vec{E}_{s}
$$

The chosen coordinate system follows the backscatter alignment convention (BSA) [5]. The coordinate system $(h, v, k)$ is right-handed and the polarization state is described in a plane orthogonal to the direction of propagation $k$. The polarization state is defined looking in the direction of propagation and the orientation angle $\theta$ (or tilt angle) is measured clockwise from the horizontal direction $h$.

An FM-CW radar needs very good isolation between the transmitter and the receiver. A possible solution is to use two antennas (transmit and receive). The antenna assembly is the major contributor to the distortion errors, and the contributions due to the different transmitting and receiving paths are negligible. With such a system, care must be taken to make the transmitting and the receiving paths practically identical, and then only antenna effects need to be considered [5]. The $-3 \mathrm{~dB}$ beam of the receiving antenna overlaps the $-3 \mathrm{~dB}$ beam of the transmit antenna at $200 \mathrm{~m}$ from the radar. The far field starts for the largest antenna at $400 \mathrm{~m}$ from DARR. When measuring in the far field, the DARR is treated like a radar of which the antenna system is reciprocal. This assumption was already made for airborne polarimetric measurements [7] and is justified when the performances of the antennas are similar or when the antenna performances are good (small channel imbalance and good cross-polarization isolation) [8]. The scattering matrix $S$ of a passive target is theoretically symmetric $\left(S_{h v}=S_{v h}\right)$. Therefore, the measured scattering matrix is symmetric, i.e., $Z=Z^{T}$, where the superscript $T$ means the transpose of the matrix. With this the calibration problem then reduces to retrieve only one antenna distortion matrix (four complex unknowns). This distortion matrix characterizes the "combined" antenna.

$$
\begin{gathered}
\left\{\begin{array}{c}
Z=Z^{T} \\
S=S^{T}
\end{array}\right. \\
\Downarrow \\
R=T^{T} \\
Z=\frac{K}{r^{2}} e^{-2 j k r} T^{T} S T .
\end{gathered}
$$

Considering one antenna distortion matrix, the transmit and the receive polarization states are modified by the same matrix $T$ in the voltage equation.

$$
V \propto\left(T \vec{e}_{r}\right)^{T} S\left(T \vec{e}_{t}\right) .
$$

The antenna distortion matrix $T$ is expressed below. $T_{h h}$ is called the field transfer function, when transmitting and receiving with horizontal polarization. $\delta_{1}$ and $\delta_{2}$ are crosstalk terms and $f$ represents the one-way copolarized channel imbalance in amplitude and phase.

$$
T=T_{h h}\left[\begin{array}{cc}
1 & \delta_{1} \\
\delta_{2} & f
\end{array}\right] .
$$

Expressing (6) in terms of components leads to the following system of equations.

$$
\left\{\begin{array}{l}
Z_{h h}=\frac{K}{r^{2}} e^{-2 j k r} T_{h h}^{2}\left(S_{h h}+2 \delta_{2} S_{h v}+\delta_{2}^{2} S_{v v}\right) \\
Z_{h v}=\frac{K}{r^{2}} e^{-2 j k r} T_{h h}^{2}\left(\delta_{1} S_{h h}+\left(f+\delta_{1} \delta_{2}\right) S_{h v}+f \delta_{2} S_{v v}\right) \\
Z_{v v}=\frac{K}{r^{2}} e^{-2 j k r} T_{h h}^{2}\left(\delta_{1}^{2} S_{h h}+2 f \delta_{1}\left(S_{1}\right) S_{h v}+f^{2} S_{v v}\right)
\end{array}\right.
$$




\section{The Rotatable Dihedral Corner Reflector A. Advantages}

One measured scattering matrix is usually not sufficient to solve the polarimetric calibration problem where four complex unknowns must be retrieved. A simple way to obtain different scattering matrices with only one reflector is to rotate the reflector around the line of sight provided that its scattering matrix differs from the identity matrix, as is the case for the dihedral corner reflector and not for a trihedral one. Experimentally, it is much easier to calibrate with one reflector where the scattering matrices are measured at the same range. Only an accurate alignment must be performed. To reduce the influence of the clutter (the calibration object is placed in a field) the backscattered signal must be high enough to guarantee a high signal to clutter ratio, keeping in mind that the size of the reflector must be reasonable (from 1 to $2 \mathrm{~m}$ ). In the hierarchy of scattering shapes, the right dihedral corner reflector has the second-strongest radar cross section [9]. The theoretical maximum value of the radar cross section of the right dihedral corner reflector is well defined and has been compared with measurements in the literature. Polarimetrically advantageous, the rotation of the dihedral around the line of sight allows one to receive a maximum voltage, for copolar measurement at angle $\alpha_{1}$ and for cross-polar measurement at angle $\alpha_{2}$. Based on measurement of several scattering matrices, the accuracy of the determination of the antenna distortion matrix can be evaluated. With all of these considerations, the rotatable dihedral corner reflector is a suitable solution for a polarimetric calibration. One drawback must be mentioned: the use of a dihedral corner demands an alignment more precise than is needed for a trihedral corner reflector.

\section{B. Practical Aspects}

The photo (Fig. 2) shows the calibration setting and Fig. 3 indicates the coordinate system of the DARR in which the dihedral corner rotates through an angle $\alpha$ in the plane $(h, v)$. The scattering matrix written in this coordinate system and the maximum radar cross section (RCS) are given below.

$$
\begin{aligned}
S(\alpha) & =\sqrt{\frac{\sigma}{4 \pi}}\left[\begin{array}{rr}
-\cos 2 \alpha & \sin 2 \alpha \\
\sin 2 \alpha & \cos 2 \alpha
\end{array}\right] \\
\sigma & =\frac{8 \pi a^{2} b^{2}}{\lambda^{2}} .
\end{aligned}
$$

The two plates forming the dihedral intersect at $90^{\circ}$. The maximum radar cross section is $38.9\left(\mathrm{dBm}^{2}\right)$ at the radar wavelength $\lambda=9 \mathrm{~cm}$ and the maximum is obtained when the direction of propagation of the incoming electromagnetic wave is perpendicular to the aperture of the corner. Thus an alignment is necessary. The angle $\alpha$ can be manually adjusted from $-90^{\circ}$ to $+90^{\circ}$ by steps of $15^{\circ}$. The support and the mount for the rotation axis are located as much as possible behind the dihedral reflector (in the

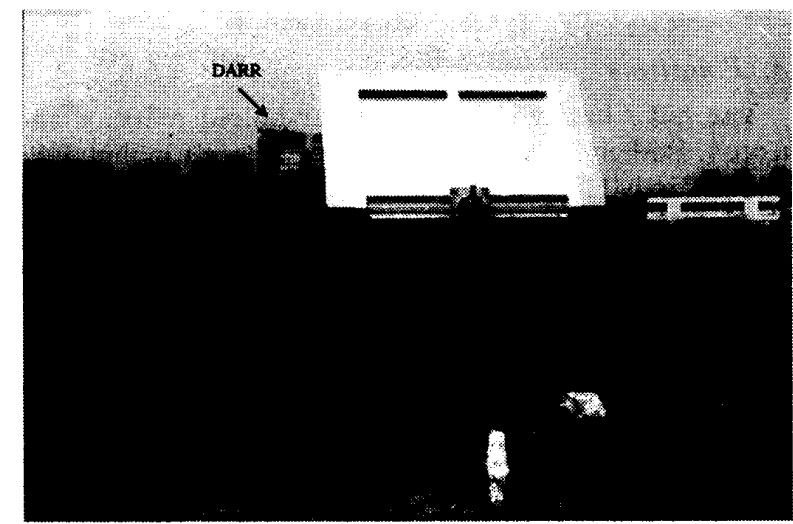

Fig. 2. The rotatable dihedral $\left(\alpha=90^{\circ}\right)$ facing the Department of Electrical Engineering building.

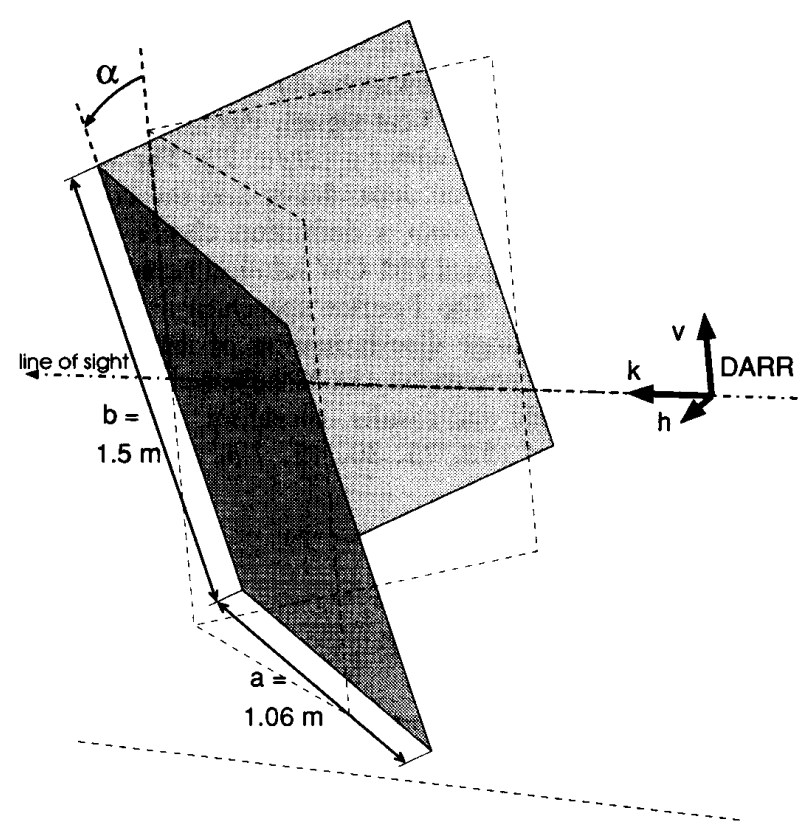

Fig. 3. The rotatable dihedral corner reflector: axes and size.

"shadow" of the reflector). The height of the reflector has been chosen as small as possible to ensure mechanical stability.

The alignment has been carried out for two positions of the dihedral $\left(\alpha=0^{\circ}\right.$ and $\left.\alpha=90^{\circ}\right)$. At $0^{\circ}$, the radar cross section tapers off gradually from the maximum with changing azimuth and sharply with changing elevation. The cross-section behavior is vice versa at $90^{\circ}$. It permits a fine alignment for both directions. The first alignment is performed in the field with an ocular pipe placed in the axis of rotation of the dihedral. Then, the radar antennas are moved in elevation and azimuth to receive maximum power. Back in the field, the dihedral is slightly moved in azimuth (with the degree of freedom built in) and elevation (adjustable feet) to optimize the received power. 


\section{DARR MEASUREMENT}

\section{A. Polarizers}

The radar has two focal-feed paraboloid reflector antennas. Both are mounted on a single antenna mount with inclined axis [1]. Two identical polarizers (each of them positioned in the feed of an antenna) can change the linear polarization at the receiving and transmitting side. The polarizers consist of a number of thin metallic vanes [2]. The bottom vane is fixed. All the other vanes can be rotated gradually and a helix of vanes results. The top vane is mechanically driven by a motor and determines the linear polarization angle at the antenna side. By using a mechanically resonant structure, the polarization angle at the top varies sinusoidally as a function of time. The selected period equals $40 \mathrm{~ms}$. Maximum polarization angle variation equals $\pm 90^{\circ}$.

\section{B. The Signal of a Nonmoving Single Target}

The received signal is down-converted with the transmitted signal to obtain the beat signal. Per sweep its formulation is given in (12) with $t \in\left[2 r_{i} / c, T\right]$. The voltage $V$ contains the polarization dependency and the complex conjugate form comes from a definition choice in [2]. Equation (13) is the typical FM-CW relation between beat frequency and range. The Fourier spectrum of the beat signal leads to the range discrimination of different targets. The frequency excursion can be adjusted at 1, 2, 5, 10,20 , and $50 \mathrm{MHz}$ and results, therefore, into DARR range resolutions of $150,75,30,15,7.5$, and $3 \mathrm{~m}$, respectively.

$$
\begin{aligned}
u(t) & \approx V^{*} e^{j\left(\omega_{i} t+\delta_{i}\right)} \\
\omega_{i} & \approx \frac{4 \pi F r_{i}}{c T} \\
\delta_{i} & \approx 4 \pi f_{c} \frac{r_{i}}{c}
\end{aligned}
$$

where

$V$ received voltage or voltage equation

$\omega_{i}=2 \pi f_{i}, f_{i}$ is the beat frequency of the target $i$

$r_{i} \quad$ range of the target $i$

$f_{c}$ center frequency $(3.315 \mathrm{GHz})$

$F$ frequency excursion

$T$ sweep time

$c$ light velocity (free space)

The received voltage is written in (15). Most of the DARR measurements are performed copolar. This means that the same polarization is used for the transmission and the reception (16). The polarization is linear and varies sinusoidally as a function of time with a period of $40 \mathrm{~ms}\left(T_{p}\right)$. Fig. 4 shows an example where 64 sweeps correspond to $40 \mathrm{~ms}$. Both simulation and measurement of the polarizers in anechoic chamber have shown that the transmitted polarization state as well as the polarization state which receives, has a polarization-dependent phase and amplitude. This dependency is weak for the amplitude (less than

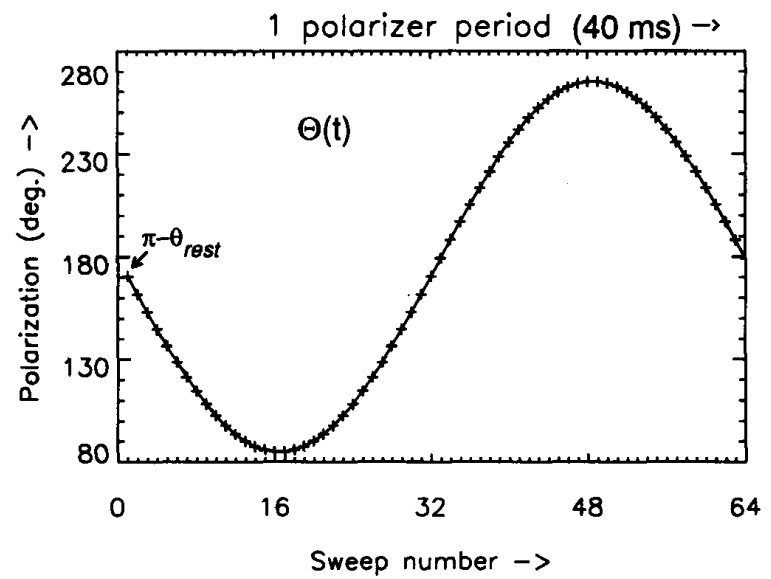

Fig. 4. Copolar measurement: the polarization (degrees) related to the horizontal (sweep time $=0.625 \mathrm{~ms}$ ).

$0.5 \mathrm{~dB}$ combining transmission and reception) and was not successfully modeled. On the other hand, there is a phase model (18) and a part of the calibration is the check of the formulation of this phase.

$$
\begin{aligned}
V= & \vec{e}_{r}^{T} Z \vec{e}_{t} \\
\vec{e}_{t}= & {\left[\begin{array}{c}
\cos \theta(t) \\
\sin \theta(t)
\end{array}\right] e^{j \varphi(t)}=\vec{e}_{r} } \\
\theta(t)= & \pi-\theta_{\text {rest }}-\frac{\pi}{2} \sin \left(2 \pi \frac{t}{T_{p}}\right) \\
\varphi(t)= & 0.45\left(\sin \left(2 \pi \frac{t}{T_{p}}\right)\right)^{2} \\
V= & \left(\cos ^{2} \theta(t) Z_{h h}+\sin 2 \theta(t) Z_{h v}\right. \\
& \left.+\sin ^{2} \theta(t) Z_{v v}\right) e^{2 j \varphi(t)} .
\end{aligned}
$$

An $N$-point DFT of the beat signal is performed per sweep. The obtained signal is a linear combination of the three elements of the scattering matrix. At least three independent measurements are necessary to retrieve the scattering matrix. To reduce noise influence on the determination of the scattering coefficients, the calculation is made over a complete polarizer period. This calculation is valid as soon as the decorrelation time of the target is much larger than $40 \mathrm{~ms}$ (a polarizer period). To give an example, the selected sweep time is often $1.25 \mathrm{~ms}$, which corresponds to 32 sweeps per polarizer period. One possible method to retrieve the scattering matrix consists of calculating the least squares solution of the set of 32 linear equations in three unknowns.

\section{The Measured Scattering Matrices of the DIHEDRAL}

The calibration measurements were performed in the summer during stable and quiet weather conditions. Two range resolutions of 15 and $30 \mathrm{~m}$ were used. This means 
a measure of the dihedral signal with two different " background" or clutter. The reliability of the data and the effect of the clutter on them can be investigated.

It is possible to change slightly the frequency excursion around the nominal positions (5 and $10 \mathrm{MHz}$ corresponding, respectively, to 30 and $15 \mathrm{~m}$ range resolutions) to get the target of interest "centered" in one range cell. There was a real-time preliminary measurement of the full spectrum of the beat signal. The polarizers were not active and stood in the "rest" position (polarization angle $\pi-\theta_{\text {rest }}$ ). From a quick-look facility, the power of the dihedral range cell was maximized. This dihedral "centering" was performed before the fine alignment of the reflector. The transmit power was $-30 \mathrm{dBm}\left(10^{-3} \mathrm{~mW}\right)$, the sample frequency was $204.8 \mathrm{kHz}$, the low-pass filter selected was $100 \mathrm{kHz}$, and the maximum range obtained was $1920 \mathrm{~m}$. Further specifications of the measurement setting are given in Table II.

Two frequency lines (also called range lines or range cells) of the spectrum of the beat signal were recorded. One contains the signal of the dihedral corner reflector and the other contains the signal of the delay line. The delay line provides the internal calibration signal of the radar. Apart from the antennas, it monitors the possible fluctuations occurring in the radar. The DARR delay line is very stable. Typically, the standard deviation is $0.1 \mathrm{~dB}$ in amplitude and $0.4^{\circ}$ in phase for a measurement time of $4.3 \mathrm{~min}$. Nevertheless, in case of any radar fluctuation, each target measurement is related to a delay line measurement. The measurement time of each orientation of the dihedral was 2 min ( 3000 polarizer periods with 32 sweeps per period) for the range resolution $15.2 \mathrm{~m}$ and 1 min (1500 polarizer periods with 64 sweeps per period) for the range resolution $30.5 \mathrm{~m}$. A coherent average is performed to obtain the mean dihedral complex signal versus one polarizer period and the scattering matrix is calculated from it. The calculated scattering matrix is the least squares solution of a set of 32 or 64 linear equations in three unknowns. The result of this processing is called the measured scattering matrix $Z$.

Figs. 5-7 give examples of the measurement of the "dihedral range cell" for one polarizer period $(40 \mathrm{~ms})$. The range resolution equals $30.5 \mathrm{~m}$. The correspondence of polarization state with sweep number is given in Fig. 4. Figs. 5 and 6 show the mean dihedral signal when $\alpha$ $=0^{\circ}$. In Fig. 5 the relative power and phase are plotted, showing both measurement and simulation. The effect of the polarization dependent phase introduced by the polarizers is clearly seen. In Fig. 6 the same measured relative phase is now plotted versus polarization and compared to the simulation without [Fig. 6(a)] and with [Fig. 6 (b)] the phase model (18). Like for the case $\alpha=0^{\circ}$, all the measurements have confirmed the validity of this phase formulation. Because the sine function describing the polarization is actually not a perfect sine, it can be seen in Fig. 6 that the expected phase jump at the polarization $135^{\circ}$ occurs a sweep later. This does not influence the calculation of the scattering matrix based on 64 data

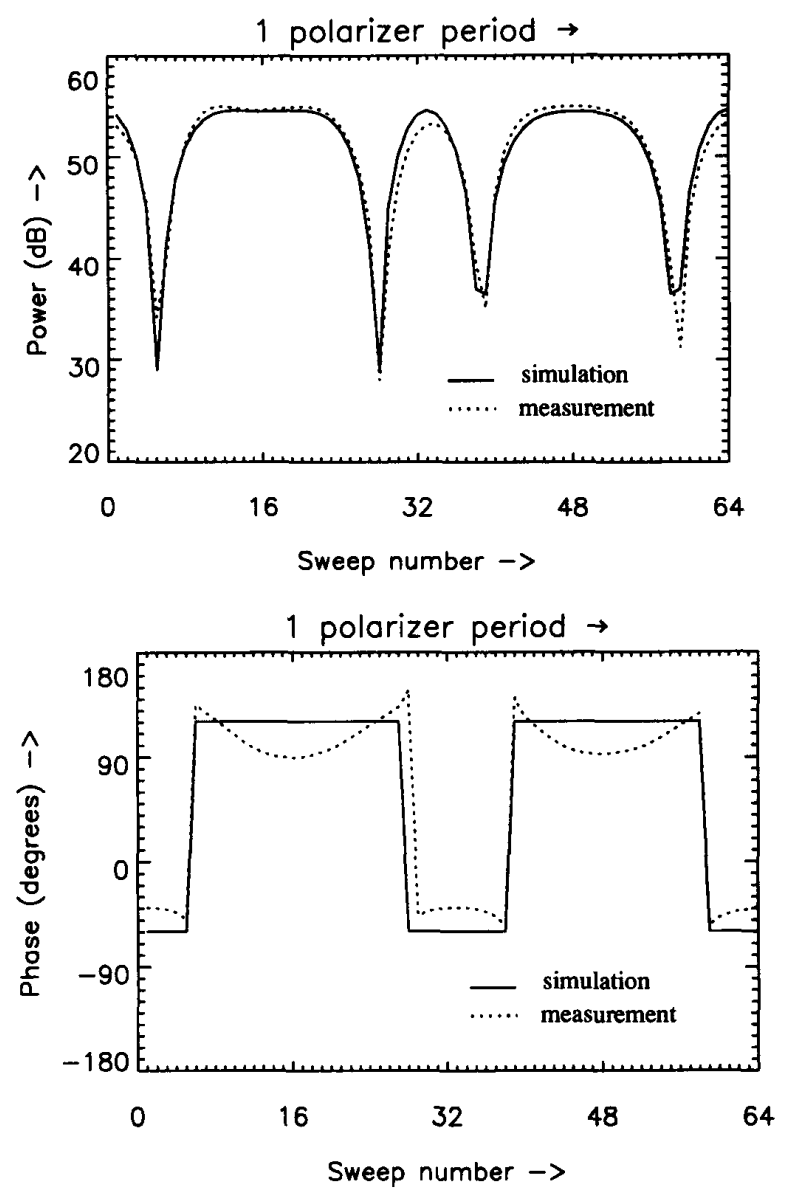

Fig. 5. Power and phase of the dihedral signal for the orientation $\alpha=0^{\circ}$.

TABLE II

Measurement Specifications. Top: Delay line. Bottom: Dihedral

\begin{tabular}{|c|c|c|}
\hline Sweep time & $1.25 \mathrm{~ms}$ & $0.625 \mathrm{~ms}$ \\
\hline Samples per sweep & 256 & 128 \\
\hline Frequency excursion & $9.88 \mathrm{MHz}$ & $4.80 \mathrm{MHz}$ \\
\hline Range resolution & $15.2 \mathrm{~m}$ & $31.2 \mathrm{~m}$ \\
\hline Delay line cell & 25 & 12 \\
\hline Range & $380 \mathrm{~m}$ & $375 \mathrm{~m}$ \\
\hline Measurement time & $8 \mathrm{~s}$ & $4 \mathrm{~s}$ \\
\hline
\end{tabular}

\begin{tabular}{|c|c|c|}
\hline Sweep time & $1.25 \mathrm{~ms}$ & $0.625 \mathrm{~ms}$ \\
\hline Samples per sweep & 256 & 128 \\
\hline Frequency excursion & $9.88 \mathrm{MHz}$ & $4.91 \mathrm{MHz}$ \\
\hline Range resolution & $15.2 \mathrm{~m}$ & $30.5 \mathrm{~m}$ \\
\hline Dihedral cell & 75 & 37 \\
\hline Range & $1139 \mathrm{~m}$ & $1130 \mathrm{~m}$ \\
\hline Azimuth resolution & $29.8 \mathrm{~m}$ & $29.6 \mathrm{~m}$ \\
\hline Measurement time & $2 \mathrm{mn}$ & $1 \mathrm{mn}$ \\
\hline
\end{tabular}




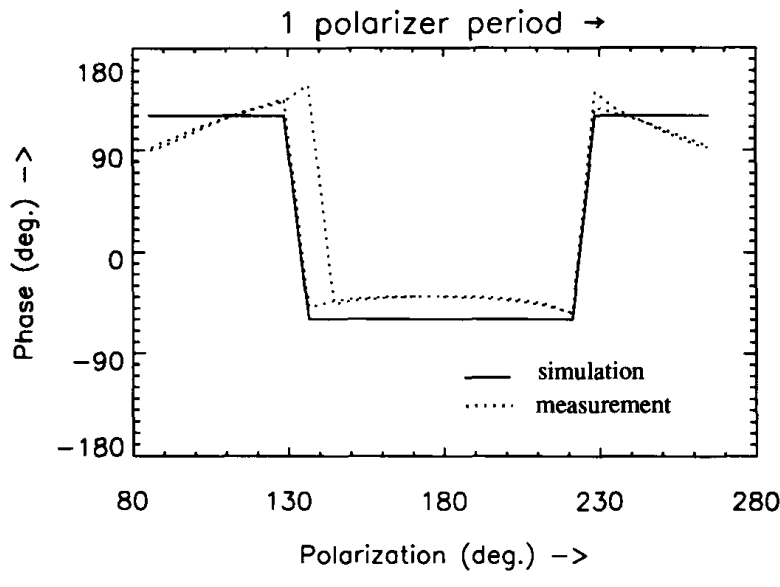

(a)

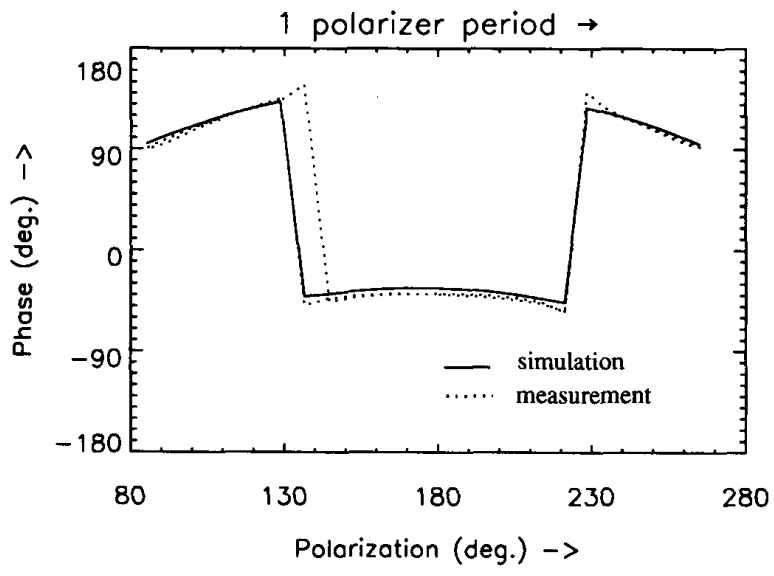

(b)

Fig. 6. The phase of the dihedral signal versus polarization $\left(\alpha=0^{\circ}\right)$ (a) without phase model, (b) with phase model.

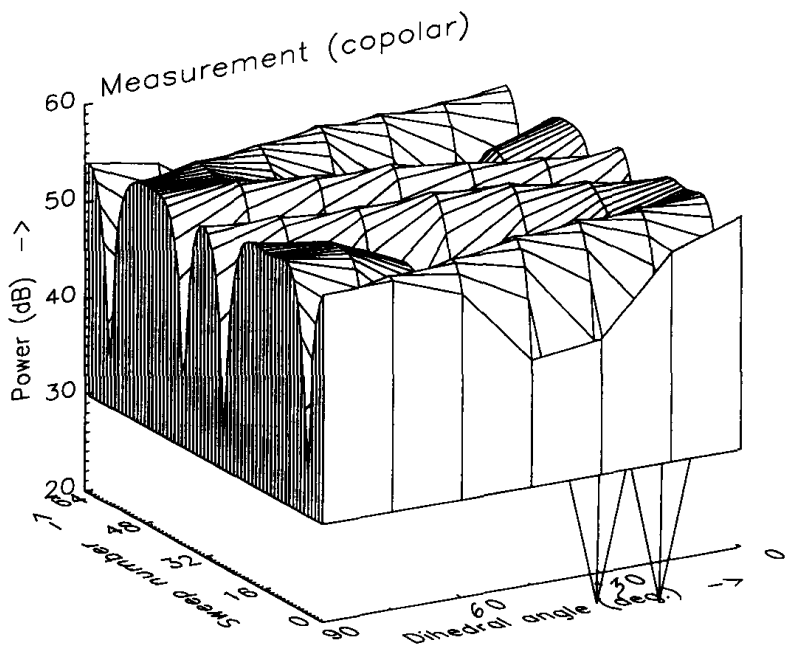

Fig. 7. The power of the dihedral signal versus time (one polarizer period) and versus several orientations of the corner reflector.
TABLE III

The Scattering Matrices of the Dihedral Rotated Through $\alpha=0^{\circ}, \alpha=15^{\circ}, \alpha=45^{\circ}$, and $\alpha=60^{\circ}$ (MEASURED AND

THEORETICAL) FOR THE 15.2 AND $30.5 \mathrm{~m}$ RANGE RESOLUTIONS

\begin{tabular}{|c|c|c|c|c|c|c|}
\hline \multirow{2}{*}{$\begin{array}{c}\text { Scattering } \\
\text { matrices }\end{array}$} & $\begin{array}{c}\text { Measurement } \\
\text { Resolution } 15.2 \mathrm{~m}\end{array}$ & \multicolumn{2}{c|}{$\begin{array}{c}\text { Measurement } \\
\text { Resolution } 30.5 \mathrm{~m}\end{array}$} & \multicolumn{2}{c|}{ Theory } \\
\cline { 2 - 7 } & $\begin{array}{c}\text { Power } \\
\mathrm{dB}\end{array}$ & $\begin{array}{c}\text { Phase } \\
\text { deg. }\end{array}$ & $\begin{array}{c}\text { Power } \\
\mathrm{dB}\end{array}$ & $\begin{array}{c}\text { Phase } \\
\text { deg. }\end{array}$ & $\begin{array}{c}\text { Power } \\
\mathrm{dB}\end{array}$ & $\begin{array}{c}\text { Phase } \\
\text { deg. }\end{array}$ \\
\hline $\mathrm{Z}_{\mathrm{hh}}\left(\alpha=0^{\circ}\right)$ & 0.0 & 0 & -0.4 & 0 & 0.0 & 0 \\
\hline $\mathrm{Z}_{\mathrm{hv}}\left(\alpha=0^{\circ}\right)$ & -38.7 & 166 & -38.8 & 158 & $-\infty$ & - \\
\hline $\mathrm{Z}_{\mathrm{vv}}\left(\alpha=0^{\circ}\right)$ & 0.4 & -181 & 0.0 & -184 & 0.0 & -180 \\
\hline $\mathrm{Z}_{\mathrm{hh}}\left(\alpha=15^{\circ}\right)$ & -1.4 & 0 & -1.6 & 0 & -1.25 & 0 \\
\hline $\mathrm{Z}_{\mathrm{hv}}\left(\alpha=15^{\circ}\right)$ & -6.3 & -181 & -6.2 & -185 & -6.02 & -180 \\
\hline $\mathrm{Z}_{\mathrm{vv}}\left(\alpha=15^{\circ}\right)$ & -0.8 & -182 & -1.2 & -183 & -1.25 & -180 \\
\hline $\mathrm{Z}_{\mathrm{hh}}\left(\alpha=45^{\circ}\right)$ & -49.3 & 0 & -31.9 & 0 & $-\infty$ & - \\
\hline $\mathrm{Z}_{\mathrm{hv}}\left(\alpha=45^{\circ}\right)$ & 0.2 & 52 & 0.0 & 63 & 0.0 & 0 \\
\hline $\mathrm{Z}_{\mathrm{vv}}\left(\alpha=45^{\circ}\right)$ & -31.4 & 30 & -32.4 & 46 & $-\infty$ & - \\
\hline $\mathrm{Z}_{\mathrm{hh}}\left(\alpha=60^{\circ}\right)$ & -5.9 & 0 & -6.4 & 0 & -6.02 & 0 \\
\hline $\mathrm{Z}_{\mathrm{hv}}\left(\alpha=60^{\circ}\right)$ & -1.1 & 0 & -1.6 & -1 & -1.25 & 0 \\
\hline $\mathrm{Z}_{\mathrm{vv}}\left(\alpha=60^{\circ}\right)$ & -5.5 & -182 & -6.2 & -183 & -6.02 & 180 \\
\hline
\end{tabular}

points. Fig. 7 gives an overview of all the measurements at the range resolution $30.5 \mathrm{~m}$. The relative power of the dihedral cell is plotted for several rotations of the dihedral. The rotation angle is related to the horizontal $\left(90^{\circ}-\right.$ $\alpha$ ), as for the polarization angle. The relative power of the noise level is $10 \mathrm{~dB}$.

Some of the scattering matrices calculated from the copolar measurements are presented in the Table III. The polarization-dependent phase introduced by the polarizers and modeled in (18) is incorporated in the calculation. The absolute phase of the scattering matrix is not considered. Therefore, the phase of $Z_{h h}$ is set to $0^{\circ}$ and the phases of $Z_{h v}$ and $Z_{v v}$ become relative. The power of the theoretical dihedral scattering matrix is normalized with respect to its maximum value. To facilitate the comparison of measurement and theory, $61.2 \mathrm{~dB}$ is subtracted from the relative power of the measured scattering matrices. Looking at the measured scattering matrices $\left(\alpha=0^{\circ}, 15^{\circ}\right.$, and $\left.60^{\circ}\right)$, the relative phase is nearer the theoretical one when using the $15.2 \mathrm{~m}$ range resolution. This resolution is experimentally found optimum for the rotatable dihedral corner. Therefore, the results of this range resolution are used to retrieve the actual scattering matrices of targets under study. The $30 \mathrm{~m}$ resolution is used for analysis of the accuracy of the calibration data. The phase model (18) significantly improves the scattering matrix results. Without the phase model, phase errors could reach $20^{\circ}$. The main correction to be performed now concerns the small channel imbalance between $h h$ and $v v$, easily noticeable in Table III at $\alpha=0^{\circ}, \alpha=15^{\circ}$, and $\alpha=60^{\circ}$. Some elements of the scattering matrices do not contain a dihedral signal like $Z_{h v}$ for the orientation $\alpha=0^{\circ}$ and both $Z_{h h}$ and $Z_{v v}$ for the orientation $\alpha=45^{\circ}$. They are a measure of the clutter and show a high signal-to-clutter ratio confirmed when measurements were performed without the dihedral corner reflector. Of course, they will not be used in the calculation of the antenna distortion matrix $T$. 
These clutter data are not accurate and cannot be interpreted further. The performed processing involving the coherent average of the signal over 1 or $2 \mathrm{~min}$ is not appropriate to treat the clutter. The purpose is to suppress as much as possible the influence of the clutter on the dihedral signal. For such a target, when the dihedral is removed the time-dependent scattering matrix must be calculated within the decorrelation time of the target and afterward quantities like $\left\langle Z_{h h} Z_{h v}^{*}\right\rangle,\left\langle Z_{h h} Z_{v v}^{*}\right\rangle$, and $\left\langle Z_{h v} Z_{v v}^{*}\right\rangle$, where \langle\rangle is a time average, can be used to describe the clutter. Another remark concerning $Z_{h v}$ for the orientation $\alpha=0^{\circ}$ and both $Z_{h h}$ and $Z_{v v}$ for the orientation $\alpha=45^{\circ}$ is that they may contain a contribution due to the dihedral imperfections if any.

\section{Polarimetric Calibration Result}

Simplifications of the system of nonlinear equations (9) in four complex unknowns $T_{h h}, f, \delta_{1}$, and $\delta_{2}$ are made. Relative phases are considered only. The phase factor $\phi$ $=2 \mathrm{kr}$ accounting for propagation between the target and the radar is neglected. The phase of $Z_{h h}$ is chosen to be $0^{\circ}$. The phases of $Z_{h v}$ and $Z_{v v}$ become relative. The same operation is carried out on the other side of the equation $\left(T^{T} S T\right)$. The receiver consists of a low-noise amplifier, a mixer, a low-pass filter to get the beat signal and a $6 \mathrm{~dB} /$ octave amplifier to compensate for the free-space expansion of radar waves for precipitation studies. The received power is proportional to $r^{-2}$ in the radar equation for volume scattering. If a group of targets such as rain drops is two times further in range, the power of their backscattered signal is $6 \mathrm{~dB}$ smaller. The consequence of this compensation for the measured scattering matrix $Z$ is described as

$$
Z=\frac{K}{r} T^{T} S T
$$

The measured scattering matrices of the dihedral contain the direct scattering from the clutter. It was not substracted even though one measurement of the clutter was performed when the dihedral was removed. A clutter measurement gives an estimate of the signal-to-clutter ratio but is not fully representative of the backscattered background signal when the dihedral signal is measured. During the campaign, the highest relative power of one element of the clutter scattering matrix is found to be 32 $\mathrm{dB}$. The lowest power of one element of the dihedral scattering matrix is $54.8 \mathrm{~dB}$. In the worse case, it means a signal-to-clutter ratio of $22.8 \mathrm{~dB}$. The average signal-toclutter ratio is estimated to be $30 \mathrm{~dB}$.

All of the measured scattering matrices of the dihedral contribute to the calculation of $T$ as soon as their elements contain a dihedral signal. These elements must be theoretically different from 0 . For the orientation $\pm 45^{\circ}, Z_{h h}$ and $Z_{v v}$ are a measure of the background scattering and only one element of the measured scattering matrix is kept for the least-squares problem: $Z_{h v}$ (modulus only). The following orientations of the dihedral were used for the calculation, $\alpha=0^{\circ}, 15^{\circ}, 30^{\circ}, \pm 45^{\circ}, 60^{\circ}, 75^{\circ}$, and $90^{\circ}$, leading to a redundant set of equations (21). $T_{h h}, f, \delta_{1}$, and $\delta_{2}$ are retrieved using nonlinear least-squares routines.

$$
\left\{\begin{aligned}
Z_{h h}(\alpha)= & \frac{K}{r} T_{h h}^{2}\left[\left(\delta_{2}^{2}-1\right) \cos 2 \alpha+2 \delta_{2} \sin 2 \alpha\right] \\
Z_{h v^{\prime}}(\alpha)= & \frac{K}{r} T_{h h}^{2}\left[\left(f \delta_{2}-\delta_{1}\right) \cos 2 \alpha\right. \\
& \left.+\left(f+\delta_{1} \delta_{2}\right) \sin 2 \alpha\right] \\
Z_{v v}(\alpha)= & \frac{K}{r} T_{h h}^{2}\left[\left(f^{2}-\delta_{1}^{2}\right) \cos 2 \alpha+2 f \delta_{1} \sin 2 \alpha\right]
\end{aligned}\right.
$$

The result of the calculation of the antenna distortion matrix is given in Table IV. Both range resolutions lead to similar results. The $15.2 \mathrm{~m}$ range resolution gives the final polarimetric calibration result. The accuracy is given in decibels for the power values and in degrees for the phase values. The accuracy is obtained comparing both results in Table IV and considering the matrices $T$ where at least one unknown was fixed, partially retrieved from each measured scattering matrix. Both range resolutions as well as individual calculation per orientation of the dihedral, give a very stable result for $T_{h h}$. Therefore, the accuracy associated to $T_{h h}$ is high $(0.2 \mathrm{~dB})$. The crosstalk terms $\delta_{1}$ and $\delta_{2}$ are equal in modulus and their phase difference is $46^{\circ}$ for both range resolutions. There is barely a phase imbalance between $h h$ and $v v\left(-1^{\circ}\right)$, since the phase model describes the phase behavior introduced by the mechanical polarizers well.

Because of the alignment radar-dihedral, $T$ characterizes the polarimetric distortion in the main direction of the antenna only. The gains $G_{t}$ and $G_{r}$ thus have their maximum values in the expression of $K$. The final formulation for DARR users is written below where $f, \delta_{1}$, and $\delta_{2}$ are the results of Table IV and $C_{h h}=5.196 \times 10^{7}$. A measurement of a target must be related to a measurement of the delay line. The value $5.196 \times 10^{7}$ is related to the delay line relative power of $43 \mathrm{~dB}$. Apart from the antennas, the delay line monitors the possible changes occurring in the radar and the processing. From it the absolute level given by $C_{h h}$ may be adjusted if necessary.

$$
\begin{aligned}
Z & =\frac{\sqrt{P_{t}}}{r} C_{h h}\left[\begin{array}{ll}
1 & \delta_{2} \\
\delta_{1} & f
\end{array}\right] S\left[\begin{array}{ll}
1 & \delta_{1} \\
\delta_{2} & f
\end{array}\right] \\
C_{h h} & =\frac{K}{\sqrt{P_{t}}} T_{h h}^{2}
\end{aligned}
$$


TABLE IV

The Antenna Distortion Matrix (15.2 and 30.5 m Range RESOLUTIONS)

\begin{tabular}{|c|c|c|c|c|}
\hline \multirow{2}{*}{$\begin{array}{c}\text { Antenna } \\
\text { distortion } \\
\text { matrix }\end{array}$} & \multicolumn{2}{|c|}{$\begin{array}{c}\text { Calibration result } \\
\text { Resolution } 15.2 \mathrm{~m}\end{array}$} & \multicolumn{2}{|c|}{$\begin{array}{c}\text { Range resolution } \\
30.5 \mathrm{~m}\end{array}$} \\
\cline { 2 - 5 } & $\begin{array}{c}\text { Power } \\
\text { (dB) }\end{array}$ & $\begin{array}{c}\text { Phase } \\
\text { (deg.) }\end{array}$ & $\begin{array}{c}\text { Power } \\
\text { (dB) }\end{array}$ & $\begin{array}{c}\text { Phase } \\
\text { (deg.) }\end{array}$ \\
\hline $\begin{array}{c}\text { Field transfer } \\
\text { function } T_{h h}\end{array}$ & $47.8 \pm 0.2$ & 0 & 47.6 & 0 \\
\hline $\begin{array}{c}\text { Cross-talk } h v \\
\delta_{l}\end{array}$ & $-43 \pm 1.5$ & $173 \pm 5$ & -39 & 166 \\
\hline $\begin{array}{c}\text { Cross-talk } v h \\
\delta_{2}\end{array}$ & $-42 \pm 1.5$ & $127 \pm 5$ & -40 & 120 \\
\hline $\begin{array}{c}\text { Channel } \\
\text { imbalance } f\end{array}$ & $0.2 \pm 0.1$ & $-1 \pm 0.5$ & 0.1 & -1 \\
\hline
\end{tabular}

TABLE $V$

The Calibrated Scattering Matrices of the Dihedral Rotated THROUGH $\alpha=0^{\circ}, \alpha=15^{\circ}, \alpha=45^{\circ}$ and $\alpha=60^{\circ}$ FOR THE $15.2 \mathrm{~m}$ RANGE RESOLUTION

\begin{tabular}{|c|c|c|c|c|}
\hline $\begin{array}{c}\text { Range } \\
\text { resolution } \\
15.2 \mathrm{~m}\end{array}$ & \multicolumn{2}{|c|}{ Calibrated data } & \multicolumn{2}{c|}{ Theory } \\
\cline { 2 - 5 } & $\begin{array}{c}\text { Power } \\
\mathrm{dB}\end{array}$ & $\begin{array}{c}\text { Phase } \\
\text { deg. }\end{array}$ & $\begin{array}{c}\text { Power } \\
\mathrm{dB}\end{array}$ & $\begin{array}{c}\text { Phase } \\
\text { deg. }\end{array}$ \\
\hline $\mathrm{S}_{\mathrm{hh}}\left(\alpha=0^{\circ}\right)$ & 28.0 & 0 & 27.9 & 0 \\
\hline $\mathrm{S}_{\mathrm{hv}}\left(\alpha=0^{\circ}\right)$ & -8.1 & -181 & $-\infty$ & - \\
\hline $\mathrm{S}_{\mathrm{vv}}\left(\alpha=0^{\circ}\right)$ & 28.0 & -180 & 27.9 & -180 \\
\hline $\mathrm{S}_{\mathrm{hh}}\left(\alpha=15^{\circ}\right)$ & 26.7 & 0 & 26.7 & 0 \\
\hline $\mathrm{S}_{\mathrm{hv}}\left(\alpha=15^{\circ}\right)$ & 21.6 & -180 & 21.9 & -180 \\
\hline $\mathrm{S}_{\mathrm{vv}}\left(\alpha=15^{\circ}\right)$ & 26.7 & -180 & 26.7 & -180 \\
\hline $\mathrm{S}_{\mathrm{hh}}\left(\alpha=45^{\circ}\right)$ & -19.0 & 0 & $-\infty$ & - \\
\hline $\mathrm{S}_{\mathrm{hv}}\left(\alpha=45^{\circ}\right)$ & 28.0 & - & 27.9 & 0 \\
\hline $\mathrm{S}_{\mathrm{vv}}\left(\alpha=45^{\circ}\right)$ & -10.1 & -165 & $-\infty$ & - \\
\hline $\mathrm{S}_{\mathrm{hh}}\left(\alpha=60^{\circ}\right)$ & 22.0 & 0 & 21.9 & 0 \\
\hline $\mathrm{S}_{\mathrm{hv}}\left(\alpha=60^{\circ}\right)$ & 26.6 & 0 & 26.7 & 0 \\
\hline $\mathrm{S}_{\mathrm{vv}}\left(\alpha=60^{\circ}\right)$ & 22.3 & -181 & 21.9 & 180 \\
\hline
\end{tabular}

TABLE VI

The Calibrated SCattering Matrices of the Dihedral Rotated THROUGH $\alpha=0^{\circ}, \alpha=15^{\circ}, \alpha=45^{\circ}$, AND $\alpha=60^{\circ}$ FOR THE $30.5 \mathrm{~m}$ RANGe Resolution

\begin{tabular}{|c|c|c|c|c|}
\hline $\begin{array}{c}\text { Range } \\
\text { resolution } \\
30.5 m\end{array}$ & \multicolumn{2}{|c|}{ Calibrated data } \\
\cline { 2 - 5 } & $\begin{array}{c}\text { Power } \\
\mathrm{dB}\end{array}$ & $\begin{array}{c}\text { Phase } \\
\text { deg. }\end{array}$ & $\begin{array}{c}\text { Power } \\
\mathrm{dB}\end{array}$ & $\begin{array}{c}\text { Phase } \\
\text { deg. }\end{array}$ \\
\hline $\mathrm{S}_{\mathrm{hh}}\left(\alpha=0^{\circ}\right)$ & 27.6 & 0 & 27.9 & 0 \\
\hline $\mathrm{S}_{\mathrm{hv}}\left(\alpha=0^{\circ}\right)$ & -8.4 & -187 & $-\infty$ & - \\
\hline $\mathrm{S}_{\mathrm{vv}}\left(\alpha=0^{\circ}\right)$ & 27.5 & -183 & 27.9 & -180 \\
\hline $\mathrm{S}_{\mathrm{hh}}\left(\alpha=15^{\circ}\right)$ & 26.4 & 0 & 26.7 & 0 \\
\hline $\mathrm{S}_{\mathrm{hv}}\left(\alpha=15^{\circ}\right)$ & 21.6 & -183 & 21.9 & -180 \\
\hline $\mathrm{S}_{\mathrm{vv}}\left(\alpha=15^{\circ}\right)$ & 26.3 & -181 & 26.7 & -180 \\
\hline $\mathrm{S}_{\mathrm{hh}}\left(\alpha=45^{\circ}\right)$ & -7.0 & 0 & $-\infty$ & - \\
\hline $\mathrm{S}_{\mathrm{hv}}\left(\alpha=45^{\circ}\right)$ & 27.8 & - & 27.9 & 0 \\
\hline $\mathrm{S}_{\mathrm{vv}}\left(\alpha=45^{\circ}\right)$ & -12.5 & 39 & $-\infty$ & - \\
\hline $\mathrm{S}_{\mathrm{hh}}\left(\alpha=60^{\circ}\right)$ & 21.5 & 0 & 21.9 & 0 \\
\hline $\mathrm{S}_{\mathrm{hv}}\left(\alpha=60^{\circ}\right)$ & 26.2 & -1 & 26.7 & 0 \\
\hline $\mathrm{S}_{\mathrm{vv}}\left(\alpha=60^{\circ}\right)$ & 21.6 & -183 & 21.9 & 180 \\
\hline
\end{tabular}

$$
\begin{aligned}
S= & \frac{r}{\sqrt{P_{t}}} \frac{1}{C_{h h}} \frac{1}{\left(f-\delta_{1} \delta_{2}\right)^{2}}\left[\begin{array}{cc}
f & -\delta_{2} \\
-\delta_{1} & 1
\end{array}\right] \\
& \cdot Z\left[\begin{array}{cc}
f & -\delta_{1} \\
-\delta_{2} & 1
\end{array}\right] .
\end{aligned}
$$

The calibrated scattering matrices of the dihedral are presented in Tables V and VI. They are compared to the theoretical scattering matrix (10). After this polarimetric calibration campaign, the relative accuracy of the scattering matrix elements is better than $0.5 \mathrm{~dB}$ and $5^{\circ}$ for the power and the phase, respectively.

\section{CONCLUSION}

A rotatable dihedral corner reflector $\left(1.5 \times 1.06 \mathrm{~m}^{2}\right)$ having a radar cross section of $38.9\left(\mathrm{dBm}^{2}\right)$ is used for the polarimetric calibration of the FM-CW ground-based radar at the Delft University of Technology. One antenna distortion matrix is calculated since the antenna system of the radar is assumed reciprocal. The copolar measurements are linear combinations of the elements of the scattering matrix. The scattering matrix is obtained with a single receive channel. Several different scattering matrices of the dihedral and two range resolutions were used to retrieve the antenna distortion matrix and the associated accuracy. The calculated antenna distortion matrix characterizes the antenna performance in the main direction. The polarimetric calibration is successful, and now the relative accuracy of the scattering matrix elements is better than $0.5 \mathrm{~dB}$ and $5^{\circ}$ for the power and the phase, respectively.

\section{ACKNOWLEDGMENT}

The authors wish to thank J. H. Zijderveld for his technical contributions to this work.

\section{REFERENCES}

[1] L. P. Ligthart and L. R. Nieuwkerk, "FM-CW Delft atmospheric research radar," IEE Proc., Pt. F, vol. 127, no. 6, pp. 421-426, Dec. 1980.

[2] L. P. Ligthart and J. S. van Sinttruyen, "FM-CW radar polarimetry," Direct and Inverse Methods in Radar Polarimetry, Part 2, W.-M. Boerner et al., Eds. Dordrecht, The Netherlands: Kluwer academic, 1992, pp. 1625-1657.

[3] H. W. J. Russchenberg, "Ground-based remote sensing of precipitation using a multipolarized FM-CW Doppler radar," Ph.D. dissertation Delft Univ., 1992.

[4] H. W. J. Russchenberg et al., "Remote sensing of precipitation with a multi-polarized Doppler (FM-CW) research radar: Application to propagation studies," $E S A J$, vol. 14, 1990.

[5] F. T. Ulaby and C. Elachi, Eds., Radar Polarimetry for Geoscience Applications. Norwood, MA: Artech House, 1990, ch. 5.

[6] A. B. Kostinski and W.-M. Boerner, "On foundations of radar polarimetry," IEEE Trans. Antennas Propagat., vol. AP-34, pp. 13951404, Dec. 1986.

[7] J. J. van Zyl, "Calibration of polarimetric radar images using only image parameters and trihedral corner reflector responses," IEEE Trans. Geosci. Remote Sens., vol. 28, pp. 337-348, May 1990.

[8] A. Freeman, "An exact solution to the problem of calibration Stokes matrix polarimetric SAR data," in Proc. IGARSS'90, vol. 1, pp. 773 777, May 1990.

[9] E. F. Knott, J. F. Shaeffer, and M. T. Tuley, Radar Cross Section Norwood, MA: Artech House, 1985, ch. 6. 


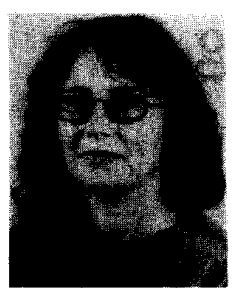

C. M. H. Unal received the D.E.A. degree in physics for remote sensing from the University of Paris, Paris, France, in 1987.

She joined the Delft University of Technology, Delft, The Netherlands, in 1988, where she works as a research scientist. She has been working on microwave remote sensing and her current research field is in radar polarimetry.

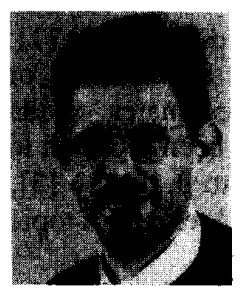

R. J. Niemeijer was born in Delft, The Netherlands, on January 23, 1964. He received the M.Sc. degree in electrical engineering from the Delft University of Technology in September 1988.

Currently, he is a research scientist working in the field of radar Doppler-polarimetry with an emphasis on real-time processing.

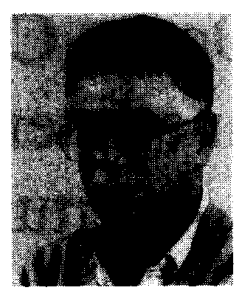

J. S. van Sinttruyen was born in Reeuwijk, The Netherlands, on June 6,1943 . He received the M.Sc. degree in electrical engineering in 1967 from the Delft University of Technology, Delft, The Netherlands.

He joined the Delft University of Technology in 1967. Since then he has been working on communication systems design and radar signal processing.

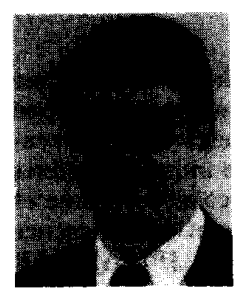

L. P. Ligthart was born in Rotterdam, The Neth erlands, on September 15, 1946. He graduated with distinction in 1969 and received the M.S. degree in electrical engineering from Delft University of Technology, Delft, The Netherlands, and the Ph.D. degree from Delft University of Technology, in 1985 .

Since 1969 he has been with the Microwave Laboratory, Delft University, working on transmission line theory, antennas, propagation, and radar. In 1988 he accepted a part-time professorship focusing on radar. In 1992 he became full-time professor in microwave transmission, remote sensing technology, and radar. 